\title{
DUNKL COMPLETELY MONOTONIC FUNCTIONS
}

\author{
JAMEL EL KAMEL AND KHALED MEHREZ
}

\begin{abstract}
We introduce the notion of Dunkl completely monotonic functions on $(-\sigma, \sigma), \sigma>0$. We establish a restrictive version of the analogue of Schoenberg's theorem in Dunkl setting.
\end{abstract}

keywords: Dunkl operators, Dunkl translation, positive definite functions, Dunkl positive definite functions, Dunkl completely monotonic functions, The Kummer confluent hypergeometric functions .

MSC (2010) 26A48, 42A82, 33C52, 42A38

\section{INTRODUCTION}

Positive definite and completely monotonic functions play an important role in harmonic analysis, for examples, in theory of scattered data interpolation, probability theory, potential theory. The most important facts about positive definite functions are the connection between positive definite and completely monotonic functions. In classical analysis a complex valued continuous function $f$ is said positive definite (resp. strictly positive definite) on $\mathbb{R}$, if for every distinct real numbers $x_{1}, x_{2}, \ldots, x_{n}$ and every complex numbers $z_{1}, z_{2}, \ldots, z_{n}$ not all zero, the inequality

$$
\sum_{j=1}^{n} \sum_{k=1}^{n} z_{j} \overline{z_{k}} f\left(x_{j}-x_{k}\right) \geq 0(\text { resp. }>0)
$$

hold true.(see [10]).

In 1930, the class of positive definite functions is fully characterized by Bochner's theorem [1], the function $f$ being positive definite if and only if it is the Fourier transform of a positive finite Borel measure $\mu$ on the real line $\mathbb{R}$ :

$$
f(x)=\int_{\mathbb{R}} e^{-i t x} d \mu(t) .
$$

In [7], we have introduced the notion of Dunkl positive definite and strictly Dunkl positive definite functions on $\mathbb{R}^{d}$. We have established the analogue of Bochner's theorem in Dunkl setting.

A continuous function $f$ on $(a, b)$ is called completely monotonic on $(a, b)$, if it satisfies $f \in C^{\infty}(] a, b[)$ and

$$
(-1)^{n} f_{1}^{(n)}(x) \geq 0
$$


for all $n=0,1,2, \ldots$ and $a<x<b$ (see [21]).

Bernstein's Theorem $[21$, p. 161], states that a function $f:[0, \infty[\longrightarrow \mathbb{R}$ is completely monotonic on $[0, \infty[$, if and only if

$$
f(x)=\int_{0}^{\infty} e^{-t x} d \mu(t)
$$

where $\mu$ is a nonnegative finite Borel measure on $[0, \infty[$.

In 1938, Schoenberg's theorem [14], asserts that a function $\varphi$ is completely monotonic on $\left[0, \infty\left[\right.\right.$ if and only if $\Phi:=\varphi\left(\|\cdot\|^{2}\right)$ is positive definite on every $\mathbb{R}^{d}$.

In this work, we introduce the notion of Dunkl completely monotonic functions on $(-\sigma, \sigma), \sigma>0$. We establish the analogue of Schoenberg's theorem in Dunkl setting. As application we study the Dunkl complete monotonicity of the Kummer confluent hypergeometric functions.

Our paper is organized as follows: In section 2, we present some preliminaries results and notations that will be useful in the sequal. In section 3, we give some properties of the Dunkl kernel, the Dunkl transform and the Dunkl translation. In section 4, we recall some results about Dunkl positive definite functions proved by the authors in [7]. In section 5, we introduce the notion of Dunkl completely monotonic functions in studying their properties, some examples are given. We state a restrictive version of Schoenberg's theorem in Dunkl setting. As application, we study the Dunkl completely monotonicity of a class of functions related to the Kummer confluent hypergeometric functions .

Let us recall some classical functional spaces :

- $C\left(\mathbb{R}^{d}\right)$ the set of continuous functions on $\mathbb{R}^{d}, C_{0}\left(\mathbb{R}^{d}\right)$ its subspace of continuous functions on $\mathbb{R}^{d}$ vanishing at infinity and $C^{\infty}\left(\mathbb{R}^{d}\right)$ its subspace of infinitely differentiable functions.

- $\mathcal{S}\left(\mathbb{R}^{d}\right)$ the Schwartz space.

- $L^{p}\left(\mathbb{R}^{d}, h_{\kappa}^{2}\right), 1 \leq p<\infty$, the space of measurable functions on $\mathbb{R}^{d}$ such that

$$
\|f\|_{\kappa, p}=\left(\int_{\mathbb{R}^{d}}|f(x)|^{p} h_{\kappa}^{2}(x) d x\right)^{\frac{1}{p}}<\infty .
$$

- Let $\sigma>0, M_{\sigma}(\mathbb{R})$ denotes the space of nonnegative finite Borel measures on $\mathbb{R}$ satisfying

$$
\int_{0}^{\infty} e^{\sigma|x|} d \mu(x)<\infty
$$

and

$$
M_{+\infty}(\mathbb{R})=\cap_{\sigma>0} M_{\sigma}(\mathbb{R})
$$




\section{NOTATIONS AND PRELIMINARIES}

Let $R$ be a fixed root system in $\mathbb{R}^{d}, G$ the associated finite reflexion group, and $R_{+}$a fixed positive subsystem of $R$, normalized so that $\langle\alpha, \alpha\rangle=2$ for all $\alpha \in R_{+}$, where $\langle x, y\rangle$ denotes the usual Euclidean inner product.

For a non zero $\alpha \in \mathbb{R}^{d}$, let use define the reflexion $\sigma_{\alpha}$ by

$$
\sigma_{\alpha} x=x-2 \frac{<x, \alpha>}{<\alpha, \alpha>} \alpha, x \in \mathbb{R}^{d} .
$$

Let $k$ be a nonnegative multiplicity function $\alpha \longmapsto k_{\alpha}$ defined on $R_{+}$with the property that $k_{\alpha}=k_{\beta}$ where $\sigma_{\alpha}$ is conjugate to $\sigma_{\beta}$ in $G$. The weight function $h_{k}$ is defined by

$$
h_{k}(x)=\prod_{\alpha \in R_{+}}|<x, \alpha>|^{k_{\alpha}}, x \in \mathbb{R}^{d} .
$$

This is a nonnegative homogeneous function of degre $\gamma_{k}=\sum_{\alpha \in R_{+}} k_{\alpha}$, which is invariant under the reflexion group $G$.

Let $T_{i}$ denote Dunkl's differential-difference operator defined in [4] by

$$
T_{i} f(x)=\partial_{i} f(x)+\sum_{\alpha \in R_{+}} \kappa_{\alpha} \frac{f(x)-f\left(\sigma_{\alpha} x\right)}{<\alpha, x>}<\alpha, e_{i}>, 1 \leq i \leq d,
$$

where $\partial_{i}$ is the ordinary partial derivative with respect to $x_{i}$, and $e_{1}, e_{2}, \ldots, e_{d}$ are the standard unit vectors of $\mathbb{R}^{d}$.

The rank-one cas: in cas $d=1$, the only choise of $R$ is $R=\{ \pm \sqrt{2}\}$. The corresponding reflexion group is $G=\{i d, \sigma\}$ action on $\mathbb{R}$ by $\sigma(x)=-x$. The Dunkl operator $T:=T_{k}$ associated with the multiplicity parameter $k \in \mathbb{C}$ is given by

$$
T_{k} f(x)=f^{\prime}(x)+k \frac{f(x)-f(-x)}{x} .
$$

Let $\mathcal{P}_{n}^{d}$ denote the space of homogeneous polynomials of degree $n$ in $d$-variables. The operators $T_{i}, 1 \leq i \leq d$ map $\mathcal{P}_{n}^{d}$ to $\mathcal{P}_{n-1}^{d}$.

The intertwining operator $V_{\kappa}$ is linear operator and determined uniquely as

$$
V_{\kappa} \mathcal{P}_{n}^{d} \subset \mathcal{P}_{n}^{d}, V_{\kappa} 1=1, \mathcal{T}_{i} V_{\kappa}=V_{\kappa} \partial_{i}, 1 \leq i \leq d
$$

According to Rösler [13], $V_{k}$ is a positive operator. De Jeu [2], prouve that $V_{k}$ is an isomorphism of $C^{\infty}\left(\mathbb{R}^{d}\right)$ whose inverse is denoted by $W_{k}$ and admit the following integral representation,

Theorem 1. For $f \in C\left(\mathbb{R}^{d}\right)$, we have

$$
V_{k} f(x)=\int_{\mathbb{R}^{d}} f(y) d \mu_{x}(y), \quad x \in \mathbb{R}^{d},
$$

where $\mu_{x}$ is a probability measure on $\mathbb{R}^{d}$ which the carrier is in the closed ball $\overline{B(0,\|x\|)}$. 
The Dunkl kernel associated with $G$ and $k$ is defined by [4]: for $y \in \mathbb{C}^{n}$

$$
\begin{gathered}
E_{k}(x, y)=V_{k}\left(e^{<., y>}\right)(x), x \in \mathbb{R}^{d} . \\
E_{k}(x, i y)=V_{k}\left(e^{<., i y>}\right)(x), x, y \in \mathbb{R}^{d} .
\end{gathered}
$$

plays the role of $e^{i<x, y>}$ in the ordinary Fourier analysis.

In the rank-one case: for the group $G=\mathbb{Z}_{2}, R e(k)>0$ we have

$$
V_{k} f(x)=\frac{\Gamma\left(k+\frac{1}{2}\right)}{\Gamma\left(\frac{1}{2}\right) \Gamma(k)} \int_{-1}^{1} f(x t)(1-t)^{k-1}(1+t)^{k} d t .
$$

In particular, for $x, y \in \mathbb{C}, \operatorname{Re}(k)>0$

$$
\begin{gathered}
E_{k}(x, y)=\frac{\Gamma\left(k+\frac{1}{2}\right)}{\Gamma\left(\frac{1}{2}\right) \Gamma(k)} \int_{-1}^{1} e^{x t}(1-t)^{k-1}(1+t)^{k} d t . \\
E_{k}(x, y)=j_{k-\frac{1}{2}}(i x y)+\frac{x y}{(2 k+1)} j_{k+\frac{1}{2}}(i x y)
\end{gathered}
$$

where for $\alpha \geq \frac{-1}{2}, j_{\alpha}$ is the normalized Bessel function.

Proposition 1. (see [11]). Let $k \geq 0$ and $y \in \mathbb{C}^{d}$. Then the function $f=E_{\kappa}(., y)$ is the unique solution of the system

$$
T_{i} f=<e_{i}, y>f, \text { for all } 1 \leq i \leq d,
$$

which is real-analytic on $\mathbb{R}^{d}$ and satisfies $f(0)=1$.

Proposition 2. (see $[6,11]$ ). For $x, y \in \mathbb{C}^{d}, \lambda \in \mathbb{C}$

(1) $E_{\kappa}(x, y)=E_{\kappa}(y, x)$

(2) $E_{\kappa}(\lambda x, y)=E_{\kappa}(x, \lambda y)$

(3) $\overline{E_{\kappa}(x, y)}=E_{\kappa}(\bar{x}, \bar{y})$

(4) $\left|E_{\kappa}(-i x, y)\right| \leq 1$

(5) $\left|E_{\kappa}(x, y)\right| \leq e^{\|x\| \cdot\|y\|}$,

\section{Harmonic analysis Related to the Dunkl operator}

In this section, we present some properties of the Dunkl transform, the Dunkl translation and the Dunkl convolution studied and developed in great detail in $[2,6,16,18]$. The Dunkl transform is defined for $f \in L^{1}\left(\mathbb{R}^{d}, h_{\kappa}^{2}\right)$ by :

$$
D_{\kappa} f(x)=c_{\kappa} \int_{\mathbb{R}^{d}} f(y) E_{\kappa}(-i x, y) h_{\kappa}^{2}(y) d y, x \in \mathbb{R}^{d} .
$$

If $\kappa=0$, then $V_{\kappa}=i d$ and the Dunkl transform coincides with the usual Fourier transform. If $d=1$ and $G=\mathbb{Z}_{2}$, then the Dunkl transform is related closely to the Hankel transform on the real line.

Theorem 2. (see [16]). 
(1) For $f \in L^{1}\left(\mathbb{R}^{d}, h_{k}^{2}\right)$, we have $D_{\kappa} f \in C_{0}\left(\mathbb{R}^{d}\right)$, and

$$
\left\|D_{\kappa} f\right\|_{C_{0}} \leq\|f\|_{\kappa, 1} \text {. }
$$

(2) When both $f$ and $D_{\kappa} f$ are $\in L^{1}\left(\mathbb{R}^{d}, h_{k}^{2}\right)$, we have the inversion formula

$$
f(x)=c_{\kappa} \int_{\mathbb{R}^{d}} D_{\kappa} f(y) E_{\kappa}(i x, y) h_{\kappa}^{2}(y) d y .
$$

(3) The Dunkl transform $D_{\kappa}$ is an isomorphism of the Schwartz class $\mathcal{S}\left(\mathbb{R}^{d}\right)$ onto itself, and $D_{\kappa}^{2} f(x)=f(-x)$.

(4) The Dunkl transform $D_{\kappa}$ on $\mathcal{S}\left(\mathbb{R}^{d}\right)$ extends uniquely to an isometry of $L^{2}\left(\mathbb{R}^{d}, h_{k}^{2}\right)$.

(5) If $f, g \in L^{2}\left(\mathbb{R}^{d}, h_{k}^{2}\right)$ then

$$
\int_{\mathbb{R}^{d}} D_{\kappa} f(y) g(y) h_{\kappa}^{2}(y) d y=\int_{\mathbb{R}^{d}} f(y) D_{\kappa} g(y) h_{\kappa}^{2}(y) d y .
$$

Let $y \in \mathbb{R}^{d}$ be given. The Dunkl translation operator $f \longmapsto \tau_{y} f$ is defined in $L^{2}\left(\mathbb{R}^{d}, h_{k}^{2}\right)$ by the equation

$$
D_{\kappa}\left(\tau_{y} f\right)(x)=E_{\kappa}(i y, x) D_{\kappa} f(x), \quad x \in \mathbb{R}^{d} .
$$

The above definition gives $\tau_{y} f$ as an $L^{2}$ function.

Let

$$
A_{\kappa}\left(\mathbb{R}^{d}\right)=\left\{f \in L^{1}\left(\mathbb{R}^{d}, h_{\kappa}^{2}\right): D_{\kappa} f \in L^{1}\left(\mathbb{R}^{d}, h_{\kappa}^{2}\right)\right\} .
$$

Note that $A_{\kappa}\left(\mathbb{R}^{d}\right)$ is contained in the intersection of $L^{1}\left(\mathbb{R}^{d}, h_{\kappa}^{2}\right)$ and $L^{\infty}$ and hence is a subspace of $L^{2}\left(\mathbb{R}^{d}, h_{\kappa}^{2}\right)$. For $f \in A_{\kappa}\left(\mathbb{R}^{d}\right)$ we have

$$
\tau_{y} f(x)=\int_{\mathbb{R}^{d}} E_{\kappa}(i x, y) E_{\kappa}(-i y, \xi) D_{\kappa} f(\xi) h_{\kappa}^{2}(\xi) d \xi .
$$

Before stating some properties of the generalized translation operator let us mention that there is an abstract formula for $\tau_{y}$ given in terms of intertwining operator $V_{k}$ and its inverse. It takes the form [18]. For $f \in C^{\infty}\left(\mathbb{R}^{d}\right)$ we have

$$
\tau_{y} f(x)=V_{k}^{(x)} \otimes V_{k}^{(y)}\left(W_{k} f(x-y)\right) .
$$

Theorem 3. (see[17]). If $\varphi \in \mathcal{A}_{k}(\mathbb{R})$, then

$$
W_{k} \varphi(x)=\frac{1}{c_{k}} \int_{\mathbb{R}^{d}} e^{i<x, y>} D_{k} \varphi(y) h_{k}^{2}(y) d y .
$$

\section{STRICTLY DUNKL POSITIVE DEFINITE FUnCTIONS}

Definition 1. A function $\varphi$ of $L^{2}\left(\mathbb{R}^{d}, h_{k}^{2}\right)$ is called Dunkl positive definite (resp. stictly Dunkl positive definte) if for every finite distinct real numbers $x_{1}, \ldots, x_{n}$, and every complex numbers $\alpha_{1}, \ldots, \alpha_{n}$, not all zero, the inequality

$$
\sum_{j=1}^{n} \sum_{k=1}^{n} \alpha_{j} \overline{\alpha_{k}} \tau_{x_{j}}(\varphi)\left(x_{k}\right) \geq 0, \quad(\text { resp. }>0)
$$


holds true. Where $\tau_{x}$ denotes the Dunkl translation.

Theorem 4. (see [7]). Let $\varphi \in \mathcal{A}_{\kappa}\left(\mathbb{R}^{d}\right)$, nonidentically zero and Dunkl positive definite function. Then $\varphi$ is strictly Dunkl positive definite.

Theorem 5. (see [7]). Let $\varphi \in \mathcal{A}_{\kappa}\left(\mathbb{R}^{d}\right)$. Then, $\varphi$ is Dunkl positive definite, if and only if, there exist a nonnegative function $\psi \in \mathcal{A}_{\kappa}\left(\mathbb{R}^{d}\right)$ such that

$$
\varphi=D_{\kappa} \psi
$$

Definition 2. A function $\Phi: \mathbb{R}^{d} \longrightarrow \mathbb{R}$ is said to be radial if there exists a function $\varphi:\left[0, \infty\left[\longrightarrow \mathbb{R}\right.\right.$ such that $\Phi(x)=\varphi(\|x\|)$ for all $x \in \mathbb{R}^{d}$.

\section{Dunkl COMPletely MONOTONiC FunCtions}

Definition 3. A function $\varphi$ is called Dunkl completely monotonic on $(-\sigma, \sigma), \sigma>0$ if $\varphi \in C((-\sigma, \sigma))$ has derivatives for all orders on $]-\sigma, \sigma[$ and

$$
(-1)^{n} T_{k}^{n} \varphi(x) \geq 0
$$

for all $n \in \mathbb{N}$ and $x \in]-\sigma, \sigma[$.

For $k=0, T_{k} f=f^{\prime}$, we retreive the classical definition.

Remark 1. It's clear that if $\varphi$ and $\psi$ are Dunkl completely monotonic, then $\alpha \varphi+\beta \psi$ is also, where $\alpha$ and $\beta$ are a nonnegative constants.

Example 1. For $y \geq 0$, the function $x \longmapsto E_{k}(-x, y)$ is Dunkl completely monotonic on $\mathbb{R}$. Indeed, for $x, y \in \mathbb{R}$ we have :

$$
E_{k}(x, y) \geq 0
$$

and

Thus

$$
T_{k} E_{k}(-x, y)=-y E_{k}(-x, y)
$$

$$
(-1)^{n} T_{k}^{n} E_{k}(-x, y)=y^{n} E_{k}(-x, y) \geq 0 ; y \geq 0 .
$$

Proposition 3. Let $0<\sigma \leq+\infty$ and $\mu$ a measure on $M_{\sigma}(\mathbb{R})$, then

$$
\varphi(x)=\int_{0}^{+\infty} E_{k}(-x, y) d \mu(y)
$$

is Dunkl completely monotonic on $[-\sigma, \sigma]$.

Proof. By example 1 and since $\mu \in M_{\sigma}(\mathbb{R})$, we get

$$
(-1)^{n} T_{k}^{n} \varphi(x)=\int_{0}^{\infty} y^{n} E_{k}(-x, y) d \mu(y) \geq 0
$$

for all $n \in \mathbb{N}$ and $x \in]-\sigma, \sigma[$. Moreover, $\varphi$ is continuous on $[-\sigma, \sigma]$, we conclude.

Proposition 4. Let $\varphi \in C^{\infty}((a, b))$ and $\varphi$ is completely monotonic function on $(a, b)$, then $V_{k} \varphi$ is Dunkl completely monotonic on $(a, b)$. 
Proof. Since $\varphi$ is completely monotonic on $(a, b)$, then

$$
(-1)^{n} \varphi^{(n)}(x) \geq 0, a<x<b .
$$

As $V_{k}$ is a positive operator and satisfies

$$
T_{k}\left(V_{k} \varphi\right)=V_{k}\left(\varphi^{\prime}\right)
$$

We get

$$
(-1)^{n} T_{k}^{n} V_{k} \varphi(x)=V_{k}\left((-1)^{n} \varphi^{(n)}(x)\right) \geq 0, a<x<b .
$$

Proposition 5. Let $\varphi \in C^{1}((a, b))$. If $\varphi$ is Dunkl completely monotonic on $(a, b)$, then $-T_{k} \varphi$ is also Dunkl completely monotonic on $(a, b)$.

Proof. Follows immediately by the definition 3 .

Theorem 6. Let $\varphi \in \mathcal{A}_{k}(\mathbb{R})$ and $\mu$ is a measure on $M_{\infty}(\mathbb{R})$ such that :

$$
\varphi(x)=\int_{0}^{\infty} E_{k}(-x, y) d \mu(y),
$$

then, the function $\varphi\left(x^{2}\right)$ is Dunkl positive definite on $\mathbb{R}$ if and only if $\varphi(x)$ is Dunkl completely monotonic.

Proof. For $t>0$, the function $x \longmapsto f_{t}(x)=e^{-t^{2} x^{2}}$ is positive definite on $\mathbb{R}$. Bochner's theorem implies that

$$
f_{t}(x)=\int_{\mathbb{R}} e^{-i t x y} d \mu(y)
$$

where $\mu$ is a finite nonnegative Borel measure on $\mathbb{R}$. Then we have

$$
V_{k}\left(f_{t}\right)(x)=E_{k}\left(-t^{2}, x^{2}\right)=\int_{\mathbb{R}}\left(\int_{\mathbb{R}} e^{-i<z t, y>} d \mu(y)\right) d \mu_{x}(z)
$$

Since the measures $\mu$ and $\mu_{x}$ are bounded, we have

$$
\phi_{t}(x)=E_{k}\left(-t^{2}, x^{2}\right)=\int_{\mathbb{R}} E_{k}(-i t x, y) d \mu(y) .
$$


Now, we will prove that the function $\phi_{t}: x \longmapsto E_{k}\left(-t^{2}, x^{2}\right)$ is Dunkl positive definite on $\mathbb{R}$, for all $t>0$. In fact from the formula (10) and theorem 3 , we have

$$
\begin{aligned}
\tau_{x} \phi_{t}(y) & =\int_{\mathbb{R}} \int_{\mathbb{R}} W_{k} \phi_{t}(\eta-\xi) d \mu_{x}(\eta) d \mu_{y}(\xi) \\
& =\int_{\mathbb{R}} \int_{\mathbb{R}} W_{k}\left(V_{k}\left(f_{t}\right)\right)(\eta-\xi) d \mu_{x}(\eta) d \mu_{y}(\xi) \\
& =\int_{\mathbb{R}} \int_{\mathbb{R}} f_{t}(\eta-\xi) d \mu_{x}(\eta) d \mu_{y}(\xi) \\
& =\int_{\mathbb{R}} \int_{\mathbb{R}^{d}} \int_{\mathbb{R}} e^{-i<t \eta, z>} e^{i<t \xi, z>} d \mu_{x}(\eta) d \mu_{y}(\xi) d \mu(z) \\
& =\int_{\mathbb{R}}\left[\int_{\mathbb{R}^{d}} e^{-i<t \eta, z>} d \mu_{x}(\eta)\right]\left[\int_{\mathbb{R}} e^{i<t \xi, z>} d \mu_{y}(\xi)\right] d \mu(z) \\
& =\int_{\mathbb{R}} E_{k}(-i t x, z) E_{k}(i t y, z) d \mu(z) \\
& =\int_{\mathbb{R}} \int_{\mathbb{R}} \overline{E_{k}(i t x, z)} E_{k}(i t y, z) d \mu(z),
\end{aligned}
$$

which implies that for every finite distinct real numbers $x_{1}, x_{2}, \ldots, x_{n}$ and every complex numbers $\alpha_{1}, \alpha_{2}, \ldots, \alpha_{n}$ not all zero, we get

$$
\sum_{j=1}^{n} \sum_{k=1}^{n} \alpha_{j} \overline{\alpha_{k}} \tau_{x_{j}} \phi_{t}\left(x_{k}\right)=\int_{\mathbb{R}}\left|\sum_{j=1}^{n} \alpha_{j} E_{k}\left(i t x_{j}, z\right)\right|^{2} d \mu(z) \geq 0 .
$$

Hence, the function $x \longmapsto \phi_{t}(x)$ is Dunkl positive definite on $\mathbb{R}$, for all $t>0$. Next, we define the function

$$
\Phi(x)=\int_{0}^{\infty} E_{k}\left(-x^{2}, t^{2}\right) d \mu(t) .
$$

Since the function $\Phi \in \mathcal{A}_{k}(\mathbb{R})$, we have for every finite distinct real numbers $x_{1}, x_{2}, \ldots, x_{n}$ and every complex numbers $\alpha_{1}, \alpha_{2}, \ldots, \alpha_{n}$ not all zero

$$
\begin{gathered}
\sum_{j=1}^{n} \sum_{l=1}^{n} \alpha_{j} \overline{\alpha_{l}} \tau_{x_{j}} \Phi\left(x_{l}\right)=\sum_{j=1}^{n} \sum_{l=1}^{n} \alpha_{j} \overline{\alpha_{l}} \int_{\mathbb{R}} E_{k}\left(-i x_{j}, \xi\right) E_{k}\left(i x_{l}, \xi\right) D_{k} \Phi(\xi) h_{k}^{2}(\xi) d \xi \\
=c_{k} \sum_{j=1}^{n} \sum_{l=1}^{n} \alpha_{j} \overline{\alpha_{l}} \int_{\mathbb{R}} E_{k}\left(-i x_{j}, \xi\right) E_{k}\left(i x_{l}, \xi\right)\left[\int_{\mathbb{R}} E_{k}(-i s, \xi) \Phi(s) h_{k}^{2}(s) d s\right] h_{k}^{2}(\xi) d \xi \\
=\int_{0}^{\infty} \sum_{j=1}^{n} \sum_{l=1}^{n} \alpha_{j} \overline{\alpha_{l}} \int_{\mathbb{R}} E_{k}\left(-i x_{j}, \xi\right) E_{k}\left(i x_{l}, \xi\right)\left[c_{k} \int_{\mathbb{R}} E_{k}(-i s, \xi) \phi_{t}(s) h_{k}^{2}(s) d s\right] \times h_{k}^{2}(\xi) d \xi d \mu(t)
\end{gathered}
$$




$$
\begin{aligned}
& =\int_{0}^{\infty} \sum_{j=1}^{n} \sum_{l=1}^{n} \alpha_{j} \overline{\alpha_{l}} \int_{\mathbb{R}} E_{k}\left(-i x_{j}, \xi\right) E_{k}\left(i x_{l}, \xi\right) D_{k}\left(\phi_{t}\right)(\xi) h_{k}^{2}(\xi) d \xi d \mu(t) \\
& =\int_{0}^{\infty} \sum_{j=1}^{n} \sum_{l=1}^{n} \alpha_{j} \overline{\alpha_{l}} \tau_{x_{j}} \phi_{t}\left(x_{l}\right) d \mu(t) \geq 0 .
\end{aligned}
$$

The last inequality holds because the function $\phi_{t}(x)=E_{k}\left(-t^{2}, x^{2}\right)$ is Dunkl positive definite and the measure $\mu$ is nonnegative. Thus the function $\Phi$ is Dunkl positive definite. Since

$\varphi\left(x^{2}\right)=\int_{0}^{\infty} E_{k}\left(-t^{2}, x^{2}\right) d \mu(t)=\int_{0}^{\infty} E_{k}\left(-t, x^{2}\right) d \mu(\sqrt{t})=\int_{0}^{\infty} E_{k}\left(-t, x^{2}\right) d \nu(t)=\Phi(x)$, we conclude.

Lemma 1. Let $\varphi \in \mathcal{A}_{k}\left(\mathbb{R}^{d}\right)$ be a radial function. If $\varphi$ is Dunkl positive definite function then $D_{k} \varphi$ is even.

Proof. For $\varphi \in \mathcal{A}_{k}\left(\mathbb{R}^{d}\right)$, we have

$$
D_{k} \varphi(x)=c_{k} \int_{\mathbb{R}^{d}} E_{k}(-i x, y) \varphi(y) h_{k}^{2}(y) d y .
$$

Thus

$$
\begin{aligned}
D_{k} \varphi(-x) & =c_{k} \int_{\mathbb{R}^{d}} E_{k}(i x, y) \varphi(y) h_{k}^{2}(y) d y \\
& =\overline{\int_{\mathbb{R}^{d}} E_{k}(-i x, y) \overline{\varphi(y)} h_{k}^{2}(y) d y} \\
& =\overline{D_{k} \varphi(x)} .
\end{aligned}
$$

Finally, corollary 1 in [7] completes the proof.

Lemma 2. Let $\varphi \in \mathcal{A}_{k}(\mathbb{R})$. If $\varphi$ is Dunkl positive definite then the function $W_{k} \varphi$ is strictly positive definite on $\mathbb{R}$.

Proof. For $\varphi \in \mathcal{A}_{k}(\mathbb{R})$, by theorem 3 , we have

$$
W_{k} \varphi(x)=\int_{\mathbb{R}} e^{i x y} D_{k} \varphi(y) h_{k}^{2}(y) d y
$$

Since $\varphi$ is Dunkl positive definite function on $\mathbb{R}$, we obtain that the function $D_{k} \varphi$ is nonnegative. Thus, for every finite distinct real numbers $x_{1}, \ldots, x_{n}$ and every complex numbers $\alpha_{1}, \ldots, \alpha_{n}$ not all zero, we have

$$
\sum_{j=1}^{n} \sum_{l=1}^{n} \alpha_{j} \overline{\alpha_{l}} W_{k} \varphi\left(x_{j}-x_{l}\right)=\int_{\mathbb{R}}\left|\sum_{j=1}^{n} \alpha_{j} e^{i x_{j} y}\right|^{2} D_{k} \varphi(y) h_{k}^{2}(y) d y \geq 0,
$$


which implies that the function $W_{k} \varphi$ is positive definite on $\mathbb{R}$. Now, suppose that the function $W_{k} \varphi$ is not strictly positive definite, then there exist distinct reals points $x_{1}, x_{2}, \ldots, x_{n}$ and complex numbers $\alpha_{1}, \alpha_{2}, \ldots, \alpha_{n}$ not all zero such that

$$
\sum_{j=1}^{n} \sum_{k=1}^{n} \alpha_{j} \overline{\alpha_{k}} W_{k} \varphi\left(x_{j}-x_{k}\right)=0 \text {. }
$$

Thus

$$
\int_{\mathbb{R}}\left|\sum_{j=1}^{n} \alpha_{j} e^{i x_{j} t}\right|^{2} D_{k} \varphi(t) h_{k}^{2}(t) d t=0 .
$$

Since $\varphi$ is Dunkl positive definite and belongs to $\mathcal{A}_{k}(\mathbb{R})$, we have $D_{k} \varphi$ is nonnegative continuous function. Then

$$
\left|\sum_{j=1}^{n} \alpha_{j} e^{i<x_{j}, t>}\right| D_{k} \varphi(t)=0 .
$$

Moreover, since $D_{k} \varphi$ is nonidentically zero, then there exist an open subset $U \subset \mathbb{R}$ such that

$$
D_{k} \varphi(t) \neq 0, \quad \forall t \in U
$$

Thus

$$
\sum_{j=1}^{n} \alpha_{j} e^{i x_{j} t}=0, \quad \forall t \in U .
$$

From lemma 6.7 in [20 p.72], we get

$$
\alpha_{j}=0, \forall j \in\{1, \ldots, n\} .
$$

Then, we deduce that the function $W_{k} \varphi$ is strictly positive definite function.

Theorem 7. Let $\varphi \in \mathcal{A}_{k}(\mathbb{R})$ be a real function and Dunkl positive definite. For $k>0$, we consider the function $\varphi_{k}(x)=D_{k} \varphi(x)|x|^{2 k+1}$. If $\varphi_{k}$ is convex and verifie $\lim _{|x| \longrightarrow \infty} \varphi_{k}(x)=0$, then

(1) $W_{k} \varphi$ is even and nonnegative.

(2) The function $\varphi(\sqrt{|x|})$ is Dunkl completely monotonic on $\mathbb{R}$.

Proof. From lemma 1, we conclude that the function $W_{k} \varphi$ is even and we have

$$
W_{k} \varphi(x)=\int_{0}^{\infty} \cos (x y) D_{k} \varphi(y)|y|^{2 k+1} d y .
$$

Since the function $\varphi_{k}(y)=D_{k} \varphi(y)|y|^{2 k+1}$ is convex downwards on $[0, \infty[$ and $\lim _{|x| \longrightarrow \infty} \varphi_{k}(x)=0$, we deduce by lemma 1 in [22], that the function $W_{k} \varphi$ is nonnegative.

By lemma 2, the function $W_{k} \varphi$ is strictly positive definite on $\mathbb{R}$, nonnegative and radial. From theorem 7.14 in [20], we conclude that the function $x \longmapsto W_{k} \varphi(\sqrt{|x|})$ 
is completely monotonic on $\mathbb{R}$. Proposition 4 completes the proof.

\section{Applications}

Theorem 8. Let $p>0$ and $k>-1$. Put

$$
\varphi_{k, p}(x)=\frac{\Gamma\left(k+\frac{1}{2}\right) e^{\frac{x^{2}}{4 p}}}{2 p^{k+\frac{1}{2}}}+\frac{\Gamma(k+1) x}{2(2 k+1) p^{k+1}} \times{ }_{1} F_{1}\left(k+1 ; k+\frac{3}{2} ; \frac{x^{2}}{4 p}\right),
$$

where ${ }_{1} F_{1}$ is the Kummer confluent hypergeometric function. Then $\varphi_{k, p}$ is Dunkl completely monotonic on $\mathbb{R}$.

Proof. Let $d \mu(t)=e^{-p t^{2}} t^{2 k+1} d t$ where $p>0$, we obtain for all $\sigma>0$

$$
\int_{0}^{\infty} e^{\sigma t} d \mu(t)=\int_{0}^{\infty} e^{t(\sigma-p t)} t^{2 k+1} d t<+\infty
$$

which implies that the measure $\mu \in M_{\infty}(\mathbb{R})$. From the Sonine formula [19, p.394], we have

$$
\int_{0}^{\infty} J_{k}(x t) e^{-p t^{2}} t^{k+1} d t=\frac{x^{k} e^{\frac{-x^{2}}{4 p}}}{(2 p)^{k+1}}
$$

where $x, p, k$ complex numbers such that $R e(p)>0, R e(k)>-1$ and $J_{k}$ stands for the Bessel function of the first kind. We change in the above Sonine formula $x$ by $i x$, we get :

$$
\int_{0}^{\infty} j_{k-\frac{1}{2}}(i x t) e^{-p t^{2}} t^{k+\frac{1}{2}} d t=\frac{\Gamma\left(k+\frac{1}{2}\right) e^{\frac{x^{2}}{4 p}}}{2 p^{k+\frac{1}{2}}}=I_{k, p}(x)
$$

On the other hand, using (4), we have

$$
\int_{0}^{\infty} E_{k}(-x, t) d \mu(t)=\int_{0}^{\infty}\left(j_{k-\frac{1}{2}}(i x t)+\frac{x}{2 k+1} t j_{k+\frac{1}{2}}(i x t)\right) d \mu(t)=I_{k, p}(x)+J_{k, p}(x),
$$

where

$$
J_{k, p}(x)=\frac{x}{2 k+1} \int_{0}^{\infty} j_{k+\frac{1}{2}}(i x t) d \mu(t)=\frac{x}{2 k+1} \int_{0}^{\infty} j_{k+\frac{1}{2}}(i x t) e^{-p t^{2}} t^{2 k+1} d t .
$$

Now, we calcul the function $J_{k, p}$. From the integral representation

$$
\int_{0}^{\infty} t^{m+1} J_{k}(x t) e^{-p t^{2}} d t=\frac{x^{k} \Gamma\left(1+\frac{m}{2}+\frac{k}{2}\right)}{2^{k+1}(\sqrt{p})^{k+m+2} \Gamma(k+1)} \times_{1} F_{1}\left(1+\frac{m}{2}+\frac{k}{2}: k+1 ; \frac{-x^{2}}{4 p}\right) .
$$

We have

$$
\int_{0}^{\infty} t^{k+m+1} j_{k}(i x t) e^{-p t^{2}} d t=\frac{\Gamma\left(1+\frac{m}{2}+\frac{k}{2}\right)}{2(\sqrt{p})^{k+m+2}} \times{ }_{1} F_{1}\left(1+\frac{m}{2}+\frac{k}{2} ; k+1 ; \frac{x^{2}}{4 p}\right)
$$


Let $m=k-1$, we obtain :

$$
\int_{0}^{\infty} t^{2 k} j_{k}(i x t) e^{-p t^{2}} d t=\frac{\Gamma\left(k+\frac{1}{2}\right)}{2(\sqrt{p})^{2 k+1}} \times{ }_{1} F_{1}\left(k+\frac{1}{2} ; k+1 ; \frac{x^{2}}{4 p}\right) .
$$

Hence

$$
J_{k, p}(x)=\frac{\Gamma(k+1) x}{2(2 k+1) p^{k+1}} \times_{1} F_{1}\left(k+1 ; k+\frac{3}{2} ; \frac{x^{2}}{4 p}\right) .
$$

Finally, by Proposition 3 we conclude that the function $\varphi_{k, p}$ is Dunkl completely monotonoic on $\mathbb{R}$.

Remark 2. For $p>0$, the function

$$
\phi_{p}(x)=\sqrt{\frac{\pi}{p}} e^{\frac{x^{2}}{4 p}}+\frac{x}{p}{ }_{1} F_{1}\left(1 ; \frac{3}{2} ; \frac{x^{2}}{4 p}\right)
$$

is completely monotonic on $\mathbb{R}$. In particular, for $p=\frac{1}{4}$, the function

$$
\begin{aligned}
\phi_{0}(x) & =2 \sqrt{\pi} e^{x^{2}}\left(1+\frac{\gamma\left(\frac{1}{2}, x^{2}\right)}{\sqrt{\pi}}\right) \\
& =2 \sqrt{\pi} e^{x^{2}}(1+\operatorname{erf}(x)) .
\end{aligned}
$$

where $\gamma(a, z)$ and $\operatorname{erf}(z)$ are respectively the incomplete gamma and error functions, is completely monotonic on $\mathbb{R}$.

\section{REFERENCES}

[1] S, Bochner, Integral transform and their application. Applied Math. Sciences 25. Springerverlag. New York, Berlin, Heidelberg, Tokyo.

[2] M.F.E. De Jeu, , Dunkl operators, Thesis Leiden University, (1994)

[3] M.F.E. De Jeu, The Dunkl transform. Invent. Math., 113 (1993), 147-162.

[4] C. F. Dunkl, Differential-difference operators associated to reflexion groups. Trans. Amer. Math. Soc., 311 (1989), 167-183.

[5] C. F. Dunkl, Integral kernels with reflexion group invariance. Can. J. Math., 43 (1991), 12131227.

[6] C. F. Dunkl, Hankel transform associated to finite reflexion groups. Contemp. Math., 138 (1992), 123-138.

[7] J. El Kamel, K. Mehrez, Dunkl positive definite functions, Tamsui Oxford Journal.

[8] A. Erdelyi, W. Magnus, F. Oberhettinger and F. Tricomi, Higher transcendental functions, volume 2, McGraw-Hill, New York, 1954.

[9] F. Hausdorff, Summationsmethoden und Momentfolgen I, Math. Z. 9 (1921), 74-109.

[10] M. Ky Fan, Les fonctions définies positives et les fonctions complètements monotones, Memorial Sciences Mathématiques, Paris (1950).

[11] M. Rösler, Dunkl operator: Theory and Applications. In orthogonal polynomials and special functions (Leuven, 2002), Vol. 1817 of lecture notes in Maths, 93-135. Springer, Berlin, (2003).

[12] M. Rösler, Generalized Hermite polynomials and the heat equation for Dunkl operators, Comm. Math. Phys. 192 , (1998) 519-542.

[13] M. Rø"sler, Positivity of the Dunkl's intertwining operator, Duke Math. J. 98 (1999), 445-463. 
[14] I.J Schoenberg, Metric spaces and completely monotone functions. Ann. Math. 39, 811841 (1938).

[15] I.J Schoenberg, Metric spaces and positive definite functions. Trans. Am. Math. Soc. 44, 522536 (1938).

[16] S. Thangavelu, Yuan Xu, Convolution operator and maximal function for the Dunkl transform, J. Anal. Math. 97 (2005), 25-55.

[17] C. Torossian, Une application des opérateurs de Dunkl au théorème de restriction de Chevalley, C.R.Acad.Sci.Paris, 318 (1994), 895-898.

[18] K. Trimèche, Paley-Wiener theorems for the Dunkl transform and Dunkl translation operators, Integral Transforms Spec. Funct. 13 (2002), 17-38.

[19] G. N. Watson, A treatise on the theory of Bessel functions (Cambridge University Press, 1944).

[20] H. Wendland , Scattered data approximations , Cambridge University Press. , Cambridge, 2005.

[21] D.V Widder, The Laplace Transform. Princeton University Press (1946)

[22] V. P. Zastavnyi, Extension of a function from the exterior of an interval to a positive-definite function on the entire axis and an approximation characteristic of the class $W_{M}^{r, \beta}$. Ukrainian Mathematical Journal, Vol. 55, No. 7, (2003).

Jamel El Kamel. Département de Mathématiques fsm. Monastir 5000, Tunisia. E-mail address: jamel.elkamel@fsm.rnu.tn

Khaled Mehrez. Département de Mathématiques iPeim. Monastir 5000, Tunisia. E-mail address: k.mehrez@yahoo.fr 